\title{
Real-time Language-Independent Algorithm for Dialogue Agents
}

\author{
Arnaud JORDAN* - Kenji ARAKI*
}

\begin{abstract}
This article presents an innovative dialogue agent designed for textual casual chatting, which can handle any language. The system acquires knowledge from a non-annotated corpus and then represents all the language aspects as a graph. Using graph traversal, the system generates one or several outputs corresponding to the user's input. Moreover, it uses graph clustering to generate word categories without using any grammar information, and finally uses these to generate more various responses. In addition, all the operations are processed in parallel, making the system able to process any input in real-time, as in human conversations. Since the system accepts any kind of input, it can also be considered to be naturally multimodal. We carried out experiments in Chinese, English, French, Japanese and Korean, and obtained results comparable to a more language-specific multilingual system. Although the current system deals only with a limited corpus and as a consequence only handles simple dialogues, we demonstrate that with further interaction and language samples it is able to adapt to more sophisticated dialogues.
\end{abstract}

keywords : natural language acquisition, language-independent system, dialogue agent, real-time, graph clustering

\section{INTRODUCTION}

Nowadays, a large number of dialogue agents have been proposed, such as ALICE [1], CSIEC [2] or Modalin [3], or are still under development. The dialogue agent research field has attracted the attention of researchers initially with the ELIZA system [4] since 1966. Some agents focus on providing pertinent information to the user, while others focus on casual chatting. The proposed system focuses on language acquisition for a dialogue agent, that is why we concentrate on casual chatting in order to acquire knowledge from the user. Since, the system acquires directly the users language (especially his native language), it does not require language expert knowledge and it is able to handle any language, making it language-independent. Moreover, after acquired enough knowledge, the proposed system could be improved to a task-oriented dialogue agent which will used the previously acquires knowledge to understand the user's commands.

With progress in research and system improve-

* Graduate School of Information Science and Technology Hokkaido University ments, dialogue agents will be able to handle more and more complicated conversation, for example the Multimodal Multi-domain Spoken Dialogue System [5] has been proposed. However, to reach this objective they use many high-level operations. Consequently, to handle these complicated processes most of these systems use very language-specific tools such as dictionaries or grammatically tagged corpora which often require domain expert knowledge. For example, many systems $[6,7]$ work only in a specific language, such as Japanese, because they use a language-specific morphological analysis tool such as JUMAN [8] to acquire a list of the words contained in the user's input. That is why such systems cannot be easily adapted to another language without a lot of work; morphological analysis tools are not available in all languages and the format of their results can be very different.

Nevertheless, some studies have aimed to develop multilingual sentence models $[9,10]$ which can suit language-independent systems. However, since languages have particular characteristics that are not common to all the languages, such models have to handle many different concepts. For example, 
the Japanese language uses particles and the English language uses articles, which are mutually not used and need specific representations. That is why the resulting models are complicated and have to be updated for each new supported language. Some methods are applicable to any language like, for example, the $\mathrm{N}$-gram [11] model, artificial neural networks [12] and the hidden Markov model [13]. However, alone they are not enough to create a flexible dialogue agent. In this paper, we attempt to combine languageindependent methods' concepts in a single graph representation in order to represent all the language characteristic using a same simple structure.

Moreover, many language-specific systems use some language-relative tricks to gain better results, such not citing the subject in Japanese sentences in order to allow the user to guess it. However, this behavior is not possible in other languages such as English. In addition, the use of language-related processing means that most systems only accept plain text input, and makes the task of developing a multimodal system complicated and time-consuming, since special algorithms have to be developed for each kind of input. In contrast, a language-independent system can handle plain text as well as any tag representing, for example, the environment of the system similarly.

Furthermore, using external language tools causes most systems to be unable to efficiently learn new expressions or frequently-used grammatically incorrect sentences. For example, if the user input the sentence "I smurf today", the verb "smurf" can be not recognized as a verb and make system enable to reply correctly. Especially, if a system use a dependency parser such as Cabocha [14], unknown words can have a big influence on the parsing result. However, a languageindependent system has no restrictions and can learn any kind of input and adapt to the user's speech, even if the input is grammatically wrong or contains new words. Consequently, the system can acquire the userspecific language in order to understand and reply correctly in future dialogue. That is why we consider that a language-independent system has important learning potential.

In this paper, we propose the framework of a dialogue agent that has been developed with the aim of handling any kind of language including languages for which no natural language processing tool is provided, which is the case of most of the languages. As a consequence, the proposed system uses no language-specific tools, such as morphological analysis tools or dictionaries, to keep the algorithm applicable to any language for which enough samples are provided. The system only uses language samples, which can be easily created automatically without requiring human task and domain expert knowledge. In order to make the system as flexible and extensible as possible, it can acquire any kind of information. Concretely, the system uses a language-independent model, i.e. a languageindependent set of links, applicable to any language in order to create a model of the language from the samples. The generated model, i.e. the generated graph depends on the target language given as input and is language-specific. Furthermore, the system handles simple dialogues and needs to be improved in future to support, for example, input that requires environmental information in order to be replied correctly such as "What time is it?" or "How is the weather?".

We hypothesize that instead of using more external tools to enhance the system, limit these tools will help the system to improve its learning component and its flexibility. Since, the external tools limitation such as language or used characters will be avoided.

It is hard to develop a language-independent dialogue agent since most of the previously-proposed methods focus on specific languages and need adaptation to be used. In addition, the results of a languageindependent system will maybe not be better than the current best systems. However, it would be useful to achieve many different objectives such as those listed below.

- Handling new terms, such as words used by young people.

- Creating a dialogue agent for minor languages for which natural language processing tools are not provided.

- Foreign language-learning using casual dialogue as training, especially for vocabulary acquisition.

- Accepting different kinds of input in order to develop a fully multimodal system.

Moreover, since the system uses no external tools, it can be easily installed on a mobile device and works 
without a network connection ${ }^{1}$. As a consequence, the system can be configured to never send data to a server to ensure the protection of the user's privacy.

Furthermore, the system could also be setup to handle nonverbal input such as sign language. Since the system can handle any kind of input such as gestures, specials tags or texts, it can be considered naturally multimodal. Another interesting exploitation of the natural multimodality of the system would be to input time tags continuously so that the system output will be influenced by these tags, for example, to make it output "good morning" in the morning and "good afternoon" after midday.

\section{ARCHITECTURE OF THE AGENT}

The proposed agent generates a graph using language samples and then uses graph traversal to generate and select the optimum responses to the user's input. As shown in Fig.1, the system does not use languageindependent information, but acquires and uses the language information, including language-specific words and grammatical constructions, using the same processing, i.e. language-independent processing, in any language in order to provide a dialogue in a specific language. The essential point of the proposed method is being able to learn any language from simple language samples, i.e. samples without languageexpert tags, language-dependent tools or annotations (like POS tags), using the same processing.

Concretely, each node of the generated graph represents a sentence such as "hello" or "I am hungry" or a part of a sentence such as "hungry" or a part of word such as "hung" and each link represent a relation between nodes. Fig.2 shows the principle of the output generation used by the system. The input of the user is compared to the input nodes which are used as entries of the graph, and then if it is similar all the related nodes' score is increased. Finally, the nodes which have the highest score are selected as outputs. The system's goal is similar to a search engine, which looks for the best pages corresponding to the user input. Therefore, the proposed approach to choose the output can also be compared to the PageRank

1 The proposed algorithm has been implemented in Java, making it directly transferable to many kind of device, such as Android one.

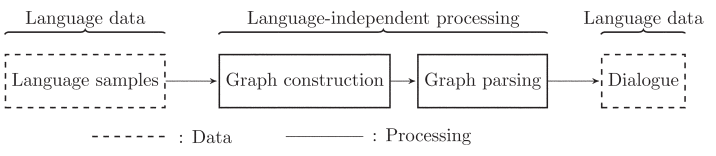

Fig.1 Language information flowchart

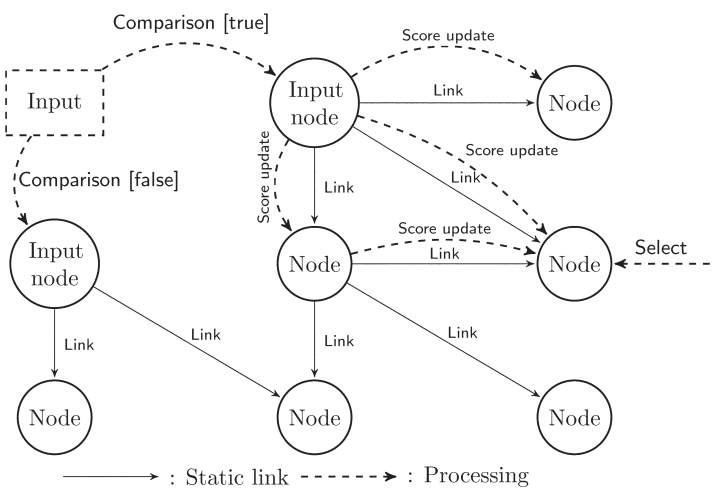

Fig.2 Principle of output generation

[15] algorithm, in which each node corresponding to a page and the related page's score is increased in function of the current pages' score.

In another way, the graph can be compared to a neural network, used for example for modeling sentences [12] ; nodes which can be used together are related by a link.

The main advantage of using graphs is to be able to adapt previously-proposed algorithms to improve the system (cf. Section 2-B5). In addition, this makes it easy to represent how the system works in a human comprehensible way, and makes the system more easily improvable.

The graph is generated using a corpus of example sentences in the target language, and the system output generation is similar to an example-base dialogue system [16]. The system looks for sentences that are strongly related to the input and then selects output from them. However, the proposed method does not use any language-expert pattern such as pattern using part of speech or category to select the output; it only uses general methods which can be applied to any language.

Consequently, as shown in Fig. 3 the system is composed of two main parts: graph construction, which replaces the use of external tools, and graph parsing, which is used to generate the system's responses.

In future development, in order to handle more complex dialogues, we will need to improve the first part to, 


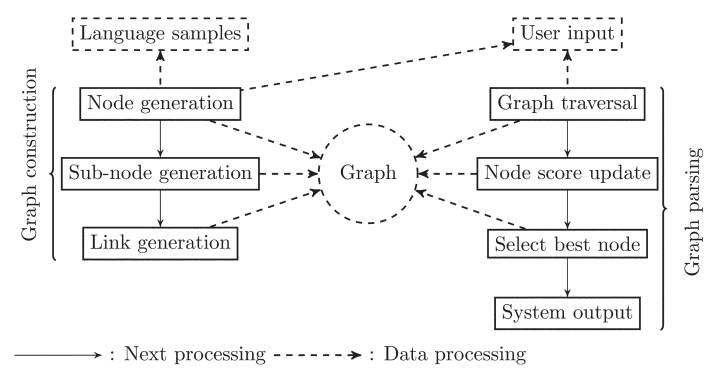

Fig.3 Flowchart of the system

for example, automatically acquire similar knowledge as knowledge presents in a lexical database such as WordNet [17].

In the next sections, we will explain in details the system. Firstly, we define the graph, i.e. nodes and links used. Then, we explain how the system generates the graph. After, we detail the link characteristics and how they are used to calculate the score of the nodes. Then, we explain how outputs are generated. Finally, we explain how all the processing have been implemented and how they are executed together in order to create a real-time dialogue.

\section{A. Definition of the graph}

All the knowledge acquired by the system is represented in a graph using nodes and directed links, as shown in Fig.4. It is important to note that this graph is a very simplified example of a graph used by the system; the graph used by the system contains many more nodes and links.

The graph construction requires only information that is present in dialogues in any language, such as sentence, part of sentence and relation between two elements. That is why the same algorithm can be used to generate graph representing any language. The used links and nodes do not represent language-

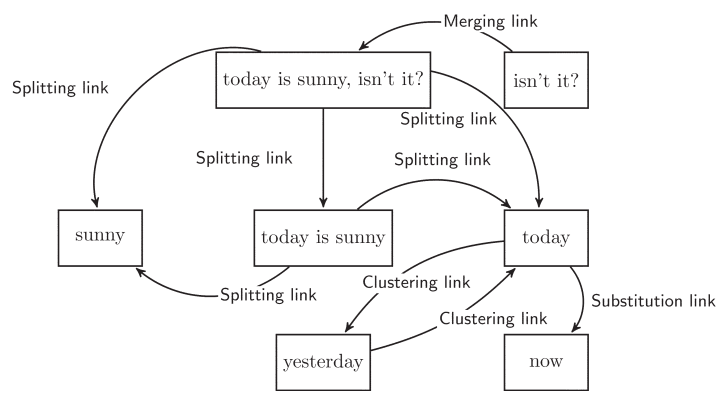

Fig.4 Example of a simple graph specific concepts such as parts of speech, but only simple relations present in any language. Concretely, the generated graph is another representation of the samples of the language, it contains the same knowledge, but is more easily usable to generate outputs to an input. It can also be considered a preprocessing of the samples in order to achieve the output generation more efficiently. The current system only acquires basic knowledge. However, the same graph can contain metadata such as time, emotions or other elements such as gestures or tasks.

\section{B. Basic links}

The system uses only basic links which are necessary for output generation [18]. These links are a representation of the structure of the sentence and a representation of the semantic relationships between words. Other kinds of link could be added in the future to improve the system output, especially to represent meta-data attached to a node.

1) Splitting link: A splitting link is generated between a node and its sub-node. Here, sub-node refers to a node which value is contained in another node value. For example, the node "I like pears" contains the nodes "I like" and "like pears", and is consequently related to it. Splitting links can be compared to the $\mathrm{N}$-gram model; however, they do not consider the order of the nodes. Splitting links are added to a node during the generation of sub-nodes (cf. Section 2-C1) .

2) Merging link: A merging link is the opposite of a splitting link; merging link and splitting links are always added together. It is set between a node and its super-node. For example, there is a merging link between "How" and "How are you?" or "How are" and "How are you?".

Specifically, a merging link is set between a sentence's part sub-node and all the complete sentences which include this sub-node. Using these links, the system can retrieve complete sentences that are eligible for output. They are generated at the same time as the splitting links, however their characteristics are different (cf. Section 2-E) .

3) Substitution link: A substitution link is provided between a node $A$ and a node $B$, if the node $B$ can be used instead of the node $A$ in the output. For example, when the user inputs "Hello", the system can answer "Hello", 
"How are you?" or "How do you feel?". As a result, there are substitution links from "Hello" to "How are you?" and to the other possible responses. We consider that a substitution links is a similar process to association in psychology [19] and it has similarities with the hidden Markov model.

However, if the input is "How are you?", the system may reply "I am fine" and "I am tired" at the same time, which would not be coherent behavior. To avoid this kind of unexpected action, it is possible in a future version of the system to implement emotional concepts; the node "I am fine" can be connected to a good emotion, i.e. a node representing this emotion and the node "I am tired" to a bad one, and then the system can be set up to output only nodes related to the emotion corresponding to its current emotional state. These emotional nodes are not related to language, since the same basic emotions are used by all humans [20]. Nevertheless, avoiding more complicated kinds of contradiction such as "I am sleeping" and "I am eating", would require many information about context to check in what condition both actions can be done and check if they are mutually compatible or not.

In addition, we also distinguish substitutions learned from the corpus called substitution sentence, and those between parts of those sentences called substitution part. We consider the first to always be correct and the second to be possibly erroneous. This distinction allows the system to define priority during the output generation.

Substitution links are not enough to be able to answer questions efficiently, as the system needs to acquire other links to know which nodes have to be deleted or replaced from the question to generate a correct response. For example, if the question is "What do you like?" the system needs to remove the question mark in the question and replace the interrogative pronoun with a noun to generate a response such as "I like chocolate”. If the system learns which parts of the question to replace and which parts to remove, it will be able to answer many similar questions.

a) Substitution removing link: In many languages, a question contains a component that indicates it to be an interrogative sentence; for example, question marks in English and French or the particle " $k a$ " in Japanese. The system needs to avoid all the nodes which contain this part in order to not always answer questions with another question. Nevertheless, the same node can be included in affirmative sentence, that is why we do not completely avoid node containing it but only decrease its score to give priority to other.

The system puts a substitution removing link on a sub-node of the question which is not present or replaced in the answer. Concretely, the link loops on the same node. For example, in the case of the tuple "It is hot, isn't it?" and "It is hot", the system puts a substitution removing link on the sub-node "isn't it?".

There are also other expressions dependent on language such as "est-ce que" in French, which serves to convert a statement into a yes-no question. These kinds of construction can be very useful for answering questions naturally. However, they are variable between languages and are hard to handle in a generic way, but with enough knowledge they can be handled by the system like other expressions.

b) Substitution replacing link: In addition, interrogative pronouns have to be replaced and never used in the answer. That is why the system puts a $s u b-$ stitution replacing link on a sub-node of the question which is replaced in the answer. This link causes the system to avoid output containing this node. For example, in the case of the tuple "What do you like?" and "I like peaches", the system puts a link on the sub-node "What". Using these links, the system can reply to questions in a natural way and avoid responses such as "I like" to the question "What do you like?".

Fig. 5 shows an example of a graph containing the different kinds of substitution link.

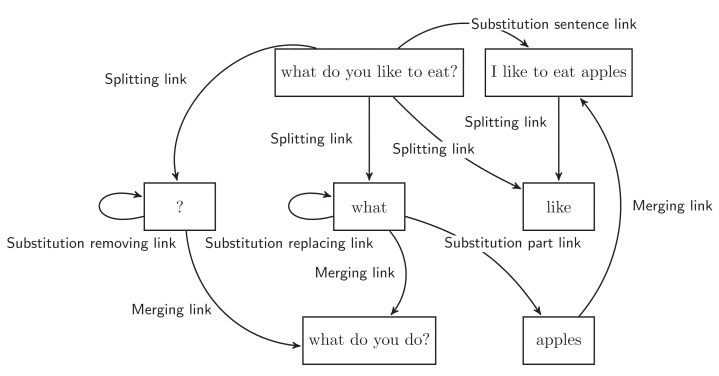

Fig.5 Example of a graph containing different substitution links

4) Association link: Association links represent the degree of similarity of two nodes. They are required to generate the clustering links. Two words 
are related if they appear in the same sentence as another word [21]. These relationships can be discovered by checking the graph structure. For example, if we take the node "I like" as a base, we can go to the sentences "I like apples" and "I like peaches" using a merging link, then using a splitting link we can go to the nodes "apples" and "pears", which can be considered as related via "I like", as both of these are "likable things".

We define the co-occurrence of words as a path \{merging link, splitting link\} in the graph. The system simply has to find this path to look for related nodes.

5) Clustering link: We use the MaxMax soft-clustering method [22] to create clusters of nodes that are related by association links. The MaxMax algorithm has been made to suit tasks such as Word Sense Induction (WSI) [23]. It is a non-parametrized and graph applicable algorithm which is very easy to implement and usable in different languages [24]. However, other clustering algorithms that work on graphs can be easily adapted to be used in the system.

The system cannot discover the word part of speech to improve the precision of clustering. However, it uses the number of links to classify nodes. For example, the node "I" has many merging links, and the node "prognostication" has only a few links. As a generalization we can hypothesize that pronouns or verbs have more links than nouns. The system only considers nodes which have a similar number of links ${ }^{2}$ to create clusters.

In addition, we had to consider the variation of the number of characters. We consider two kinds of language: those with a small number of different characters where each word contains several letters, like French and English, and those with a high number of different characters where many words are composed of only one or two characters, like Japanese and Chinese. The number of substrings is $n(n+1)^{2}(n$ is the number of characters), and consequently more a language uses long strings, the more sub-nodes may be generated (cf. Section 2-C1) and the more chances the system has to make mistakes while clustering.

Fig. 6 shows an example of two possible clustering

2 Two nodes which have a number of links different from more than $30 \%$ are not put in the same cluster. For example, a node which has 100 links and a node which has 131 links are not put in the same cluster. results for words related to "I eat". We expect the second result to generate correct outputs.

I eat \{"apples", \{"apples"'s sub-nodes: "appl", "ap", ... $\}, \ldots\}$

I eat $\{$ "apples", "peaches", "pears", ... $\}$ (expected)

Fig.6 Example of two possible clustering results

\section{Graph generation}

The system uses training samples of the targeted language (cf. Section 4) to generate the first nodes of the graph. Firstly, each sentence present in the samples is converted into a node called an input node. For example, the sentences "Hello" and "How are you?" are converted into two distinct nodes. Then, the system also converts the user inputs into new input nodes. For example, if the user input "I like spring”, a new node containing this sentence will be generated.

The generated graph contains the language information, and as a consequence is language-dependent, i.e. a graph generated with English samples contains English sentences, whereas if we use Japanese samples, it will contain Japanese sentences. Nevertheless, since the generation method and the exploitation of the graph are language-independent, the proposed algorithm is applicable to any language.

\section{1) Sub-node generation}

In natural language processing, one of the most common tasks is morphological analysis. However, in the context of a multilingual system we cannot use tools such as ChaSen [25] which are only available for some languages. A solution would be to use unsupervised word segmentation [26]. However, we need a real-time and fast adaptive algorithm and consequently, we had to develop our own sentence tokenizer algorithm to be able to split a sentence ${ }^{3}$.

This is why we simply use already existing nodes to try to split new ones. For example, the system will use the node "I like" to split the node "I like peaches" into "I like" and "peaches".

The generated sub-nodes can represent several words, e.g. "like peaches", as well as a single word like "peaches" or a part of a word like "ach". This behavior can be useful for handling determiners such as "a" or "my"; for example, "a cat" and "my cat"

3 Spaces are not used to split sentence, since they are not commonly used in all the languages. 
are different things and can be represented by different nodes.

This method can generate some noise, i.e. nodes that are not useful for output generation, as well as useful ones. For example, in the experiment, the sentence "where is your friend?" has seven splitting links, "you”, "where is", "where”, "is your friend?", “your friend?", "r friend?" and "where is you". About $2 \%$ of generated nodes contain a part of a word in English. However, as has been proven for stochastic resonance [27], noise could also help the system to generate correct and useful responses. Specifically, the system may access ineffective nodes which will not be used to generate the output of the system, because they are regularly related to all the other nodes and will not increase the score of a particular node. As a result, their influence on the choice of the output is limited. However, too numerous links make the system slow, that is why we consider forget useless links.

\section{New node inferring}

Using the previously-created clustering links, the system is able to create new sentences by substituting a node with another node in the same cluster. For example, if the starting corpus contains the sentence "There is a dog" and the system creates the cluster \{“dog", "cat", "rabbit"\}, it can generate the new sentences "There is a cat" and "There is a rabbit". We can compare this process to the crossover in genetic algorithms. This is a kind of reflection to infer new knowledge from existing knowledge. In general, the system uses previously acquired language samples to select an output. However, in order to create more various outputs, we consider that a dialogue agent must be able to also generate new sentence in order to provide more precise output to the user and to adapt its output to the input more precisely.

\section{E. Link characteristics and node score calculation}

Each kind of link between nodes has its own characteristics. These are used during graph traversal to calculate the node's score and the link's cost.

- Node score denotes the importance of a node. This value is used to select the best node to output.

- Link cost refers to how much power is needed to traverse the link and go to the next node. This value is used to limit the graph traversal.

All links that have a link cost exceeding a defined value are ignored by the system.

Each kind of link has the following three characteristics.

- Weight denotes the importance of the linked node; links that reference directly a possible answer such as a substitution link have a high value.

- Distance denotes the difference between two nodes. Splitting links only remove a part of the information; consequently their distance is small, contrary to substitution link which can point to a totally different node.

- Base cost is used to calculate the cost of the link.

Changing these characteristics will change the system's behavior. For example, we can make the system generate more sentences ${ }^{4}$, but these will not all be correct. Alternatively, we can make the system take more careful behavior ${ }^{5}$ and only output sentences that are definitely correct.

We use Equation (1) to calculate the node score, and the weight and distance are calculated by aggregating the total value of the links used to arrive at this node from the user's input. Nodes which score is higher than zero are called active nodes.

We chose this equation in order to increase score and then to select nodes that are different from the input, but which are related to it, i.e. the system must avoid outputting the same sentence as the input. In detail, the more nodes are followed, the more the weight and the distance increase. This is why the exponential function is used to limit the selected nodes to the vicinity of the input node. For example, we want to avoid selecting nodes that have a high weight, but which are quite far from the input and consequently, potentially inadequate as output. If the input is "What do you eat?" the response "I eat bread" must be selected but the response to the latter "Do you like bread?" must be avoided since it is too far from the input and the user may not understand

4 Decrease the distance value or the base cost.

5 Increase the base cost. 
the relation with the input.

$S_{n}=\frac{\sum \text { weight }}{e^{\sum \text { distance }}}$

- $S_{n}$ is the score of a specific node.

For example, if the node $A$ is related by splitting, substitution part and merging links to the input, then its score will be increased by $(2+5+3) / e^{0.75+2+1}=0.24$.

In addition, we use Equation (2) to calculate the link cost. The base cost is defined for each kind of link. However, in order to decrease the influence of very frequent sub-nodes we use the number of links in a similar way to the tf-idf method [28]. Specifically, the more links a node has, the more the cost of each link increases. The objective here is to limit the use of nodes such as "are" or "do" which are present in many sentences. In addition, we use a logarithm to reduce the difference between two nodes that only have a small difference in number of links, and consequently can be considered similar.

$C_{l}=c \times\left(1+\log \left(n_{\text {link }}\right)\right)$

- $C_{l}$ is the cost of the link.

- $c$ is the base cost of the target link type.

- $n_{\text {link }}$ is the number of links of the corresponding type from the same node.

Table 1 contains the empirically defined values for each type of link. In detail, these values have been chosen after many manual attempts to reach the optimal compromise between the number and correctness of the output. The association and clustering links are used to create new nodes, but are not used during graph traversal, i.e. during the output generation, and as a result do not have specific characteristics.

Table 1 LINK CHARACTERISTICS

\begin{tabular}{l|ccc}
\hline Link & Cost & Distance & Weight \\
\hline Splitting & 1.50 & 0.75 & 2.00 \\
Merging & 0.99 & 1.00 & 3.00 \\
Substitution sentence & 2.51 & 2.00 & 20.00 \\
Substitution part & 2.50 & 2.00 & 5.00 \\
Substitution removing & 2.50 & 1.00 & -8.00 \\
Substitution replacing & 1.00 & 1.00 & -10.00
\end{tabular}

\section{F. Output generation}

The system updates score of nodes in function of the input, and then selects the highest ones as outputs.

In order to update the score of all the nodes related to the user input, the system accesses the graph by all the input nodes at the same time and looks for all the nodes that match, include or are included in the user's input, if it is the case the node score is increased. Then, all the related nodes score is also increased in function of the followed links. Since only input nodes which are similar to the input are used to enter in the graph, even if the graph is composed of a huge number of nodes, only a part of them will be visiting to generate outputs. Fig.7 shows the score update

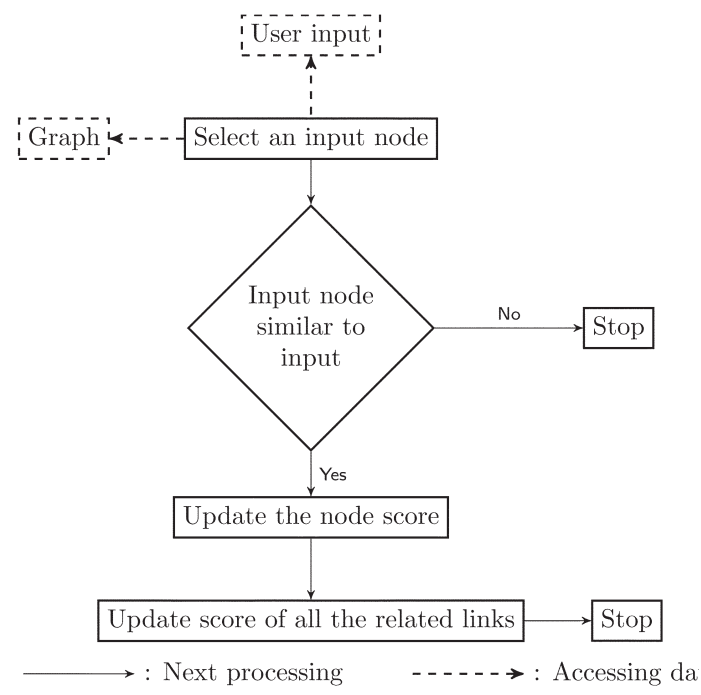

Fig.7 Flowchart of the score update

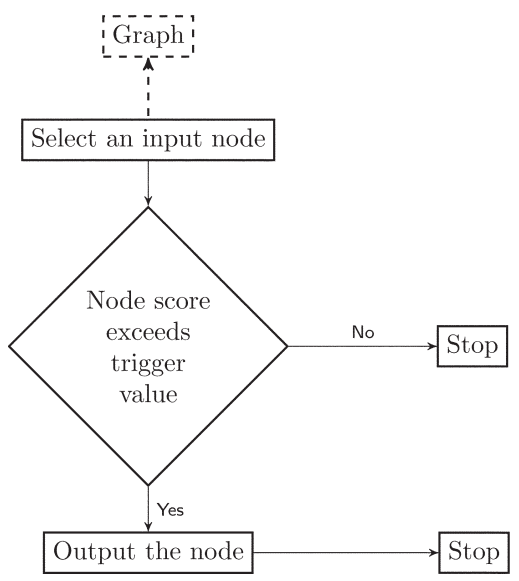

Fig.8 Flowchart of the output selection 
flowchart.

Then, at the same time, the system periodically (every $200 \mathrm{~ms}$ in the experiment) checks the graph input nodes to look for nodes which score exceed a trigger value (cf. Section 3-I2), then outputs the sentences represented by the nodes to the user. Fig. 8 shows the node selection flowchart. Since input nodes represent only complete sentences, the system never outputs incomplete sentences.

For example, if the input sentence is "I like making cookies", the nodes "I like" and "cookies" are included in it, and their score will be increased. Both of these are related to the node "I like eating cookies" by a merging link, and its score will be increased too. If the score of the node exceeds the trigger value, the system will output "I like eating cookies".

If no node's score exceeds the trigger value after the entire graph has been traversed the system will output an apology sentence corresponding to a void tag (cf. Section 3-I1) such as "I am sorry, I cannot reply".

1) Example of output generation: Fig.9 shows an example of output generation. In this example, the input is "now, what do you eat?" and the system outputs two sentences "I eat salad" and "I do not eat cookies". Since the first sentence is closer and its score is higher than the second, the system will output it first. The second response can be considered unnatural, but the system would need more knowledge such as meta-data tags to avoid replying responses that are not totally coherent. We can also note that

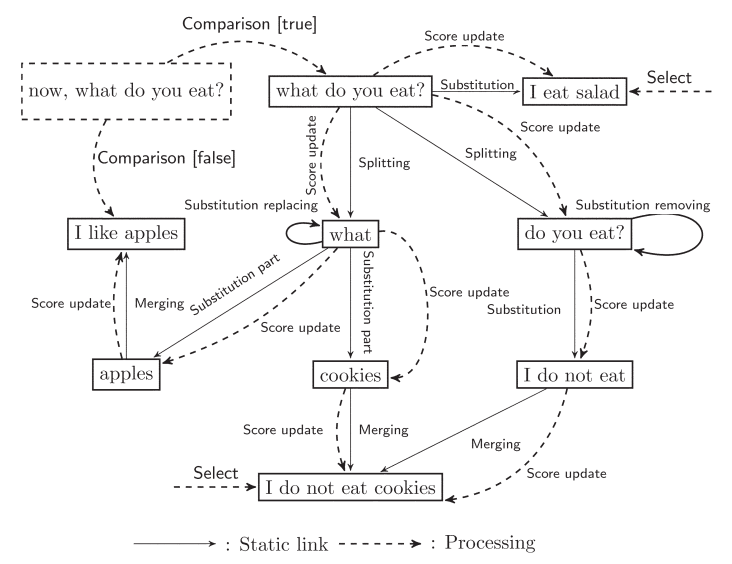

Fig.9 Processing of user's input the score of the input nodes "do you eat?", and "I do not eat", "I like apples" increase too, and may be output in function of the parameters of the system.

2) Example of mistake handling: Using the previously-explained links the system is able to handle some simple input mistakes such as misspelling. Concretely, the input string is split into many different substrings, and some of these substrings contain errors, whereas the others are correct and can be used to retrieve a correct string. Fig. 10 shows an example of a mistake that the system is able to handle.

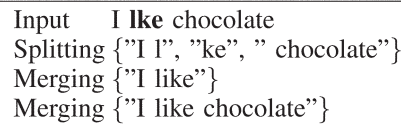

Fig.10 Example of handling of misspelling

In the example, the substring "I l" and "ke" are related to the same string "I like" by a merging link, and since they are contained in it, the score of the node "I like" increases too. Then, because both "I like" and "chocolate" point to the node "I like chocolate", this node's score is also increased. Finally, the score of the correct sentence "I like chocolate" is increased and the node will be used to generate a response.

It is also important to note that this kind of mistake generally appears when the user uses a keyboard and not a voice input interface.

\section{IMPLEMENTATION OF THE SYSTEM}

A dialogue is a real-time process [29], to enable the system to receive input at any time we implement each operation of the graph construction and parsing in a different thread which are all executed in parallel. Here, real-time means that the system accepts asynchronous input and the user does not have to wait for system output to input a new sentence. All the inputs change the structure and the active nodes of the graph, and then influence the output generation.

Fig.11 shows the hierarchy of the threads presents in the system. Each thread can access the graph to read or update it.

In order to avoid using all the processing resources each thread is periodically executed. Table 2 shows the period of execution of each thread, these values can be adapted to the performance of the machine 


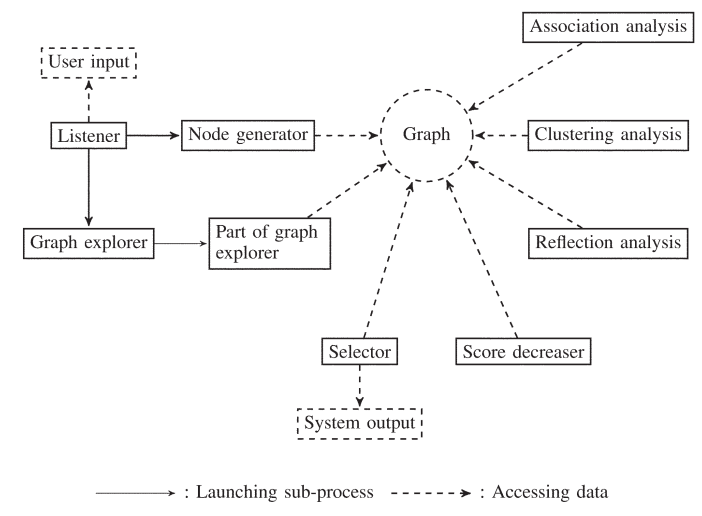

Fig.11 System's threads hierarchy

Table 2 THREADS PERIOD

\begin{tabular}{l|c}
\hline Thread & Period [ms] \\
\hline Listener & 0 \\
Selector & 200 \\
Score decreaser & 2000 \\
Association analysis & 600 \\
Clustering analysis & 600 \\
Reflection analysis & 600
\end{tabular}

which executes the system. The listener is always waiting user input and as result has a period of $0 \mathrm{~ms}$.

\section{A. Association analysis}

The association analysis thread contains all the processes used to add association links between nodes present in the graph. Specifically, the thread updates the association links of the active nodes using algorithm of Fig.12. The input of the algorithm is a node of the graph and it generates association links related to it.

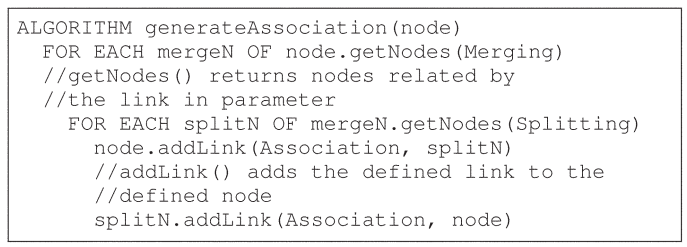

Fig.12 Algorithm used to generate association links

\section{B. Clustering analysis}

The clustering analysis thread contains all the processes used to add clustering links in the graph. The system updates the clustering links of the active nodes, in order to update the links of nodes that may be used for the output generation.

\section{Reflection analysis}

In the reflection analysis thread the system generates new possible sentences using the clustering links (cf. Section 2-D). Concretely, the system attempts to generate new sentences containing an active node. For example, if the node "rice" is active, the system will attempt to generate new sentences using the nodes of the same cluster as "rice", such as "bread", to generate new sentences such as "I bought rice" from the original sentence "I bought bread".

Fig.13 shows the algorithm used to infer new sentences. The input of the algorithm is an active node of the graph which may replace a node from the same cluster in an already existing input node. The generated new node is added to the graph in order to be used during the output generation.

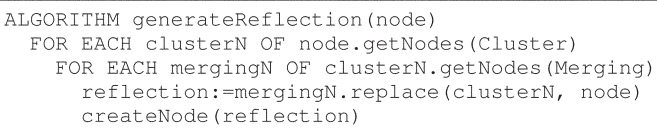

Fig.13 Algorithm used to infer new sentence

\section{Listener}

The listener thread simply waits for the user input. When there is an input it will launch the node generator and graph explorer thread, to generate the responses corresponding to the input.

\section{E. Node generator}

The node generator thread uses the input to enrich the graph used by the system. It uses the input to add new nodes including input nodes and links into the graph. In addition, the same thread is used to import a corpus into the system.

Fig.14 shows the function called when the system adds a new node in the graph. Each time a new sentence is inputted, the system will create a new input node, and then check whether it can be used to split previously acquired sentences, i.e. input nodes. The input of the algorithm is a sentence or a tag from the user or from languages samples. This input is firstly encapsulated (using the generateNode () function) into a node, in order to be compared with already existing node, if it already exists no processing is executed. 


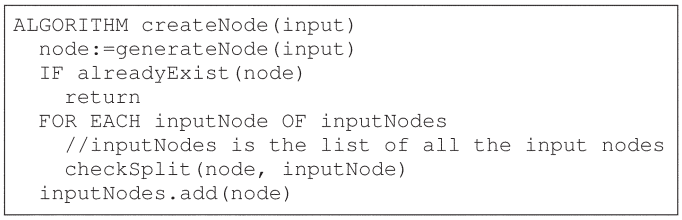

Fig.14 Function called when the user inputs a sentence

Fig.15 shows the function called to check whether a node can split another and vice versa. The system checks whether a node contains or is contained in the other one, and if this is the case it splits the containing node by the contained node. In addition, the system will also check whether any sub-node can be used to split the newly-created node.

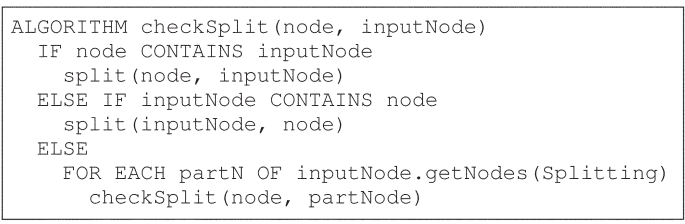

Fig.15 Function used to check whether a node can split an other

Fig.16 shows the function called to split and to set the required splitting and merging links between two nodes.

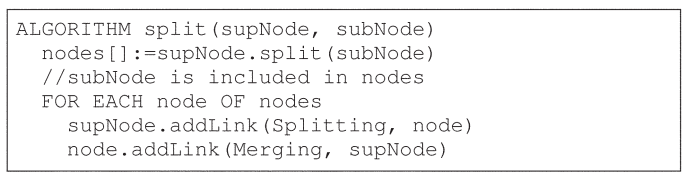

Fig.16 Function used to add a new sub-node to the graph

In addition, this thread will also generate substitution links between nodes it generates. Fig.17 shows the algorithm used to create substitution links between a question and its response. The two parameters, question and response, are nodes representing a part of dialogue of the targeted language samples. Concretely, a substitution link is set between an utterance and its response, and a substitution part links is set between all the sub-nodes of the utterance and all the nodes of the response which are not present in the utterance. Substitution replacing links and substitution removing links are also generated when the question contains nodes which are not present in the reply, and as a consequence has to be replaced or removed to select a correct output.

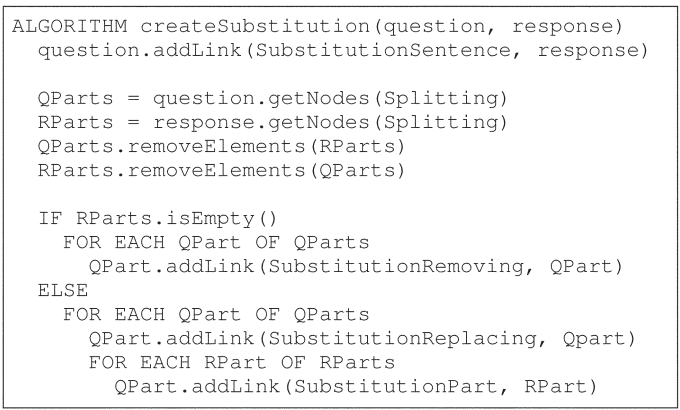

Fig.17 Function called to create substitution links

\section{F. Graph explorer}

The graph explorer thread starts a part of graph explorer thread on all the input nodes of the graph. As a result, the graph is accessed by several agents at the same time. This parallel processing improves the speed of the output generation.

\section{G. Part of graph explorer}

The part of graph explorers look for nodes that match, include or are included in the input, starting with an input node.

As shown in Fig.18, the algorithm takes the user input as parameter, then it is firstly encapsulated into a node to be compared with each input node of the graph, in order to look for all the nodes that match, include or are included in the user' s input ${ }^{6}$; all of these nodes' scores will be increased.

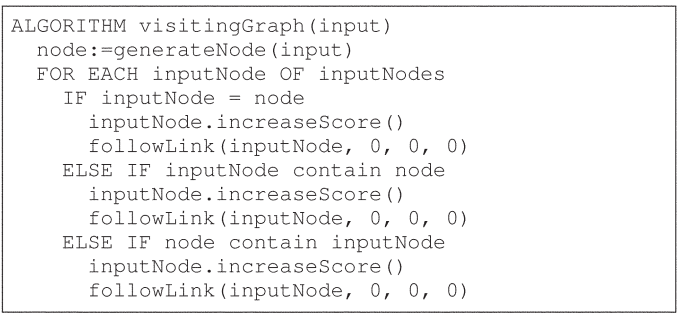

Fig.18 Algorithm used to find nodes related to the input

Then, as shown in Fig.19, using the previously acquired links, the score of all the nodes that are related to a matching input node will be increased too in function of their links' characteristics. All the nodes will be accessed until the link cost exceeds

6 For example, the node "I like eating" includes "eating" and is included in "I like eating chocolate". As a result, these two nodes' scores are increased. 
a defined value, i.e. $M A X C O S T$ (set to 5 in the experiment). The link cost of each link is added to the previous link cost, and as a result the cost used in the comparison increases each time the system follows a link.

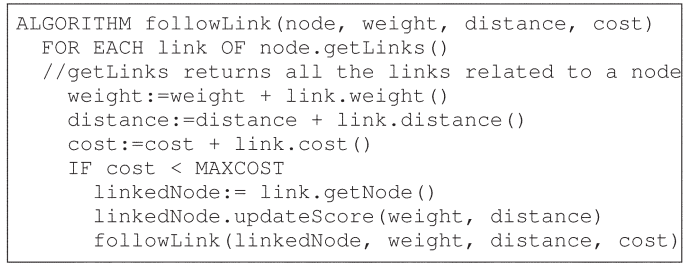

Fig.19 Algorithm used to update score of the node

\section{H. Score decreaser}

Since the system increases nodes score, it is also necessary to decrease score in order to not output node which was in relation with previous inputs.

The score decreaser thread causes the score of all the active nodes to fall to zero after a defined time which vary in function of the node score (about 5 seconds in the experiment). Concretely, the node scores are periodically updated using Equation 3. The score of each node is divided by a coefficient (set to 2 in the experiment) in order to decrease its score in function of its current value. In addition, we removed one in order to be sure that the score will tend to be a negative value and, as a result that all the nodes are set to be inactive if there is no input during some time.

$S_{t}=\frac{S_{t-1}}{k}-1$

- $S_{t}$ is the node's score at time $t$.

- $k$ is used to determine the decrease order.

Precisely, the score value is continuously brought closer to zero using a unique parameter, and when it becomes zero, the node is set inactive.

\section{Selector}

The selector thread looks for nodes for which their score exceeds the current trigger value (cf. Section 3I2). In addition, it also updates the current trigger value in function of the time and the system's previous output.

As shown in Fig.20, the node that has the best score, which exceeds the trigger value, and which is a complete sentence, is selected and output to the user. The algorithm does not require any parameter, since it only checks the graph state to look for possible outputs. Outputted node score is set to zero to not output several times the same sentence.

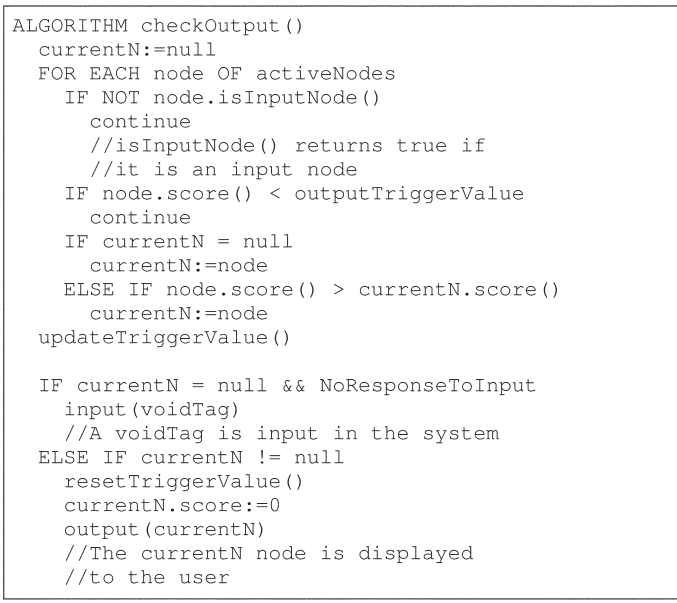

Fig.20 Algorithm used to generate output

1) VoidTag: If the system finished to explore the graph and no node can be outputted to reply to the user input (NoResponseToInput), then it will input a voidTag in the system exactly like user sentences are input.

Some dialogue samples provided to the system as basic knowledge contain this tag and the corresponding response, and consequently the system will be able to provide an output to it such as "Sorry, I cannot answer". Precisely, the void tag is substituted, using a substitution link, with an apology sentence to indicate to the user not to wait for any other output.

2) Output trigger value: In order to create a realtime system, the system has to reply in a minimum amount of time like a human would, but with maximum relevance, i.e. with the best possible response. In order to produce this kind of behavior, the system uses a dynamic trigger representing the minimal score required by a node to be an output candidate. The initial value is set in function of the system state and then the value of the trigger decreases in function of the time spent, using Equation 4. Consequently, the system searches first for nodes that have a high score, and the more time passes, the more it searches for lower-scoring nodes, since the trigger value becomes 
lower.

$V_{t}=V_{i-1}-t \times k$

- $V_{i}$ is the initial value.

- $t$ is the time since the initial value.

- $k$ is used to determine the decrease order.

This equation makes the trigger value decrease using a single parameter that is empirically set (set to 2 in the experiment). The parameter can be changed to make the system output more or less sentences for each input.

Fig. 21 shows an example of the trigger changes.

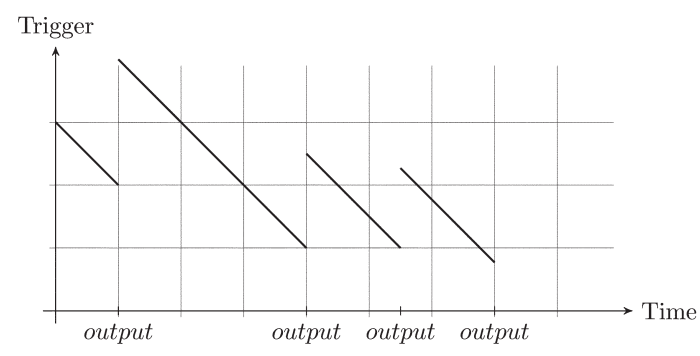

Fig.21 Example of trigger changes

After the system selects an output, the trigger value is reinitialized. This value is calculated using Equation 5. Concretely, the system uses the mean of the last five output values $(n=5)$ multiplied by $2(m$ $=2$ ) to calculate the new trigger value. This method allows the system to adapt to the nodes' scores automatically.

$$
V_{i}=\left(\sum_{k=i-n}^{i-1} V_{k}\right) / n \times m
$$

- $V_{i}$ is the value of the trigger at time $i$.

- $i$ is the output number.

- $n$ is the number of previous output used.

- $m$ is used to scale the trigger value.

\section{TRAINING SAMPLES}

To generate the graph, the system uses two kinds of basic resources, dialogue samples and knowledge samples. They contain no grammar information and need no complex creation processes. Compared to a common system based on AIML [30] database, the corpus of the presented system contains no tags and all the rules are automatically acquired from the samples. For example, wild-cards, which are often present in corpus-based chat-bots, are not present in the samples. They have to be statistically induced ${ }^{7}$ by the system.

Samples are different for each language and can contain particular characteristics not common to all languages. For example, although metaphors are widely understandable between different cultures [31] ( such as "time is money", "love is madness", etc) there are expressions rooted in culture. For instance, raccoon dog in Japanese can indicate a cunning person, while in Western cultures this animal has no such connotations. However, since the graph generated from samples and from user's inputs is languagespecific; the language model adapts to the user. Therefore, the system will not output utterance "he is like a raccoon dog" in English since it is unlikely to learn it from the user, but it could be learned from a conversation in Japanese. In addition, the lack of distinct difference between single and plural in Japanese is not a problem since the samples and the user's inputs will be adapted to it. For example, number can be defined by specific expressions or by context using relations to other nodes. Even if these expressions are specific to one language they are represented within the same language-independent set of links.

Moreover, it is also possible to create samples which contain only tags indicating sentiment or environment context and which could be used in different languages. For example, the positive sentiment of "good" can be related to "sunny weather".

In detail, the sentences of the samples are used as arguments of previously-presented functions such as createSubstitution () or createNode ().

1) Dialogue samples: The dialogue samples contain some very simple dialogues such as those shown in Fig.22, used to acquire substitution and splitting links in the target language. They can be, for example, extracted from a dialogue between two humans or from screenplays.

7 A word that has many substitution links can be considered to be a kind of wild-card. 
U1: What do you drink?

U2: I drink milk

Fig.22 Example of a dialogue sample

a) Language differences: Each language has its own way to express some casual questions. For example, during the experiments subjects used four forms of asking someone's age in Japanese, two in French and one in English. Fig.23 contains some questions asked to the system by the user in different languages.

- $\quad$ Ni duodale (zh) How old are you? (formal)

- $\quad$ Ni ji suile $(z h)$ How old are you? (informal)

- How old are you? (en)

- Tu as quel age? ( $f r$ ) How old are you? (informal)

- Quel age as-tu? (fr) How old are you? (formal)

- Nan-saidesu ka (ja) How old are you? (formal)

- Ikutsudesu ka (ja) How old are you? (informal)

- Nenrei o oshietekudasai (ja) Tell me your age (formal)

- Toshi wa? (ja) What's your age? (informal)

- Myeoch sal-ieyo (ko) How old are you? (informal)

- Neo myeoch sal-iya (ko) How old are you? (formal)

zh: Chinese ${ }^{a}$, en: English, fr: French, ja: Japanese, ko: Korean ${ }^{a}$ Mandarin Chinese

Fig.23 Different ways to ask one's age in different languages

A dialogue system has to be flexible enough to reply to all of these different forms using limited samples. In order to handle this problem, the proposed framework can, for example, use the sub-node "myeoch sal" of the Korean sentence "Myeoch salieyo" to output the response to this sentence when the user inputs "Neo myeoch sal-iya" too. Consequently, the system can correctly output many variations of questions that are not present in the samples, using sub-nodes of questions that are in the samples.

2) Knowledge samples: The knowledge samples are a list of simple sentences such as "I like ice-cream" or "The president of the USA is Obama".

The knowledge samples are used to increase the possible outputs of the system. These samples can be collected easily, since they consist of a list of simple sentences that are not contextually related. They can be collected from any text such as a Wikipedia article, a book or from users' inputs.

\section{EXPERIMENTS}

In order to check the validity of the proposed method, we carried out output correctness experiment. We used the same protocol as the evaluation of generality of SeGa-ILSD [32]. We choose this baseline since it focuses on casual dialogues and has been adapted and evaluated in several languages. However, for our system we do not use an ELIZAtype system to generate part of the responses as did the baseline, since we consider it is too languagedependent. We also do not use morphological analysis tools as the baseline system does, the generated subnodes are used instead (cf. Section 2-C1).

In order to be able to carry out a correct comparison with the baseline, we used voice recognition as the input method. However, it is important to note that voice input methods use a huge amount of languagerelated resources and are only available for a small number of languages. As the presented system is text-oriented, during the experiments voice input is simply used instead of a keyboard.

We use the Google speech recognition implemented in an Android ${ }^{8}$ application to acquire the user's input. This recognition tool uses a speaker-independent GMM-HMM model ${ }^{9}[33]$.

We asked subjects to evaluate the system's responses as below, where an example response is provided for each choice.

- Correct reply Meaning is correct, and expression is natural.

- Example What do you like?: I like playing basketball.

- Semi-correct reply Meaning is correct, but expression is not natural.

Example What do you like?: I like play basketball.

- Erroneous reply Meaning is not correct.

Example What do you like?: Apple are ta.

The aim of the evaluation is to check whether the system's responses are grammatically correct and correspond to the user input. Nevertheless, we asked the subjects to evaluate the system's response as erroneous if the system does not reply to the input question. For example, if the input is "What will you do tomorrow?" and the response is "I don't know", it is considered to be erroneous even if the output is grammatically correct and a human could reply in such

8 http://developer.android.com

9 Gaussian Mixture Models-Hidden Markov Models 
a way. Since, this response is provided when no node matches the input.

We define the system precision as the rate of responses that are not erroneous.

\section{A. Baseline}

We used the SeGA-ILSD system as a baseline for this experiment. This spoken dialogue system uses an inductive learning method based on genetic algorithms with sexual selection. Concretely, it acquires rules automatically from pairs consisting of an utterance and its associated response, and attempts to do a crossover of two rules to create a new one. Rules that generate erroneous output are progressively removed from the system using user feedback.

In order to do a crossover of two rules, the system needs to identify each word in the sentences, for which it uses a morphological analysis tool ${ }^{10}$.

In addition, when no rules are found to reply to the input the system uses an ELIZA-like system to generate the output. The ELIZA-like system contains manually created rules that are different for each language.

Moreover, the baseline uses Microsoft Japanese recognizer (Version 6.1), Microsoft English Recognizer (Version 5.1) and Microsoft Simplified Chinese Recognizer (Version 5.1) as speech recognition tools ${ }^{11}$.

\section{B. Experiment preparation}

We asked three native speakers each of Chinese, English, French, Japanese and Korean to imagine a simple casual dialogue of about 40 sentences in order to create the dialogue samples.

The Japanese knowledge samples were directly extracted from a previous research [36]. For this research subjects were asked to teach some common knowledge to a spoken dialogue agent. The same samples were also manually translated into the four other languages by a native speaker.

For Chinese (Mandarin) we used simplified Chinese characters. We did not make any distinction

10 JUMAN Version 5. for Japanese, Apple Pie Parser Version 5.9 [34] for English and ICTCLAS for Chinese [35].

11 The version 6.1 stems from Microsoft Office 2003 and the version 5.1 is extracted from the package Microsoft Speech SDK 5.1: http://www.microsoft. com/en-us/download/ details.aspx?id $=10121$ between different kinds of English or French.

1) Splitting parameter: To avoid the generation of many nodes ${ }^{12}$ in languages using Latin characters, we set a minimal character length of four to split a string in English and French. Moreover, we set a minimal length of two ${ }^{13}$ for Korean.

We also did not use capital letters, in order to increase the node matching rate. For example, in the sentences "Cats are cute" and "I like cats", "Cats" and "cats" are the same word, but they will be considered different by the system because of the capital letter. However, we kept meaningful capital characters, such as in the case of proper nouns.

It is important to note that the knowledge required to know whether a word needs a capital letter or not depends on the language. With a bigger corpus, we think this task can be avoided without significant impact to the system, since the number of nodes will be sufficient to split all the words with and without a capital letter.

Characters depend on the language, but they do not make the system language-dependent. The user can input any character into the system; the output generation process will not be affected. For example, a word such as "t $\% \& 3=\mathrm{f}$ " can be learnt by the system in the same way as all other words.

\section{Experiment settings}

We asked each subject to evaluate approximately 50 output sentences by the system. We also gave instructions to each subject for them to input only simple, complete and grammatically correct sentences into the system. Concretely, most of the subjects input some greetings and simple questions about the system, such as its name or age, and some more general questions such as "What are you doing?"

The steps of the experiment are listed below.

1) Choose the language.

2) Enter a name.

3 ) Enter a sentence using voice recognition.

4) Evaluation of voice recognition (erroneous recognition results are also input in the system).

12 A node for many short strings that can make the system slower and use memory.

13 Hangeul characters are composed of 24 consonant and vowel letters in groups of two, three or four. 
5) Evaluation of responses to the input sentence.

6 ) Return to step 3.

Table 3 shows information about the experiment subjects.

\section{Table 3 SUBJECT'S INFORMATION}

\begin{tabular}{l||ccccc|c}
\hline & Zh & En & Fr & Ja & Ko & Total \\
\hline \hline Subject & 7 & 4 & 4 & 13 & 5 & 33 \\
\hline Male & 2 & 2 & 4 & 5 & 1 & 14 \\
\hline Female & 5 & 2 & 0 & 8 & 4 & 19 \\
\hline Student & 7 & 2 & 2 & 9 & 5 & 25 \\
\hline Worker & 0 & 2 & 2 & 4 & 0 & 8 \\
\hline Age [years] & 21.9 & 21.5 & 30.0 & 23.1 & 22.0 & 23.1
\end{tabular}

Table 4 summarizes information about the system knowledge. The number of nodes refers to the number of nodes created before the user starts the dialogue. To count the number of words, we simply split words using spaces for English and French, for Japanese we used the JUMAN morphological analysis tool, for Chinese we use Google Translator' ${ }^{14}$ word segmentation, and for Korean we used the HanNanum tools [37]. In addition, for Korean, we also checked the result using Google Translator's word segmentation, obtaining 1,296 words. The number of dialogues corresponds to the number tuples question/ response taught to the system.

Table 4 CORPUS INFORMATION

\begin{tabular}{l|rrrrr}
\hline Number & \multicolumn{1}{|c}{ Zh } & \multicolumn{1}{c}{ En } & \multicolumn{1}{c}{ Fr } & \multicolumn{1}{c}{ Ja } & \multicolumn{1}{c}{ Ko } \\
\hline of nodes & 1410 & 715 & 839 & 1623 & 664 \\
of words & 1246 & 1029 & 1343 & 1268 & 708 \\
of characters & 1818 & 4853 & 7465 & 2514 & 3383 \\
of sentences (knowledge) & 100 & 100 & 100 & 100 & 100 \\
of dialogues (dialogues) & 88 & 69 & 102 & 85 & 107
\end{tabular}

\section{Experiment results}

1) Speech recognition precision: The speech recognition does not recognize punctuation. Consequently, subjects were not able to input commas or question marks.

The precision is calculated using Equation $6^{15}$.

$$
P=\frac{n_{c}}{n_{c}+n_{i}}
$$

- $P$ is the precision.

- $n_{c}$ is the number of correct recognitions.

- $n_{i}$ is the number of incorrect recognitions.

This formula is the same as that used in the baseline. We prefer it to the word error rate (WER)

14 https://translate.google.com/

15 We use the common precision formula [38] value in terms of capability to carry out a correct comparison with the baseline.

Table 5 shows the precision achieved for each language during the experiment.

Table 5 SPEECH RECOGNITION PRECISION

\begin{tabular}{c|ccccc|c}
\hline & Zh & En & Fr & Ja & Ko & Mean \\
\hline $\begin{array}{c}\text { Precision } \\
{[\%]}\end{array}$ & 93.4 & 86.7 & 88.0 & 86.7 & 91.7 & 89.3
\end{tabular}

2) Evaluation of responses: Fig.24 shows an example of dialogue with the system. Evaluations by the subject for each output are presented in parentheses.

User: How old are you?

System: I am one year old (Correct reply)

User: What is your favorite food?

System: I like spring (Erroneous reply)

System: I want to eat fried foods (Semi-correct reply)

User: I like fried foods too

System: I want to eat fried foods (Correct reply)

System: I can eat spicy foods (Semi-correct reply)

Fig.24 Example of dialogue with the proposed system

We can notice that the responese "I like spring" was evaluated erroneous by the subject. The system had no sample similar to "What is your favorite food?", and as result, outputs the node "I like spring" which was related to the sub-node "favorite" of the input. The semi-correct response "I want to eat fried foods" was selected from the sub-node "food".

Some subjects also input more complex sentences, for example, a subject inputted the sentence "okay how about we meet on Friday night at 7 p.m." and the system replied using previously acquired sentence "Yes I am free on Friday nights" which was evaluated semi-correct.

Table 6 contains the experiment results compared to the baseline [39]. For the baseline, the values in parentheses correspond to the evaluation results for the ELIZA system's outputs. All the baseline results were obtained in a single evaluation run.

\section{E. Analysis of results}

It is clear that the results are similar in the five evaluated languages, and that they are superior to the baseline results if we do not count ELIZA's responses. In addition, as the results of the baseline are quite different for each language, we consider 
Table 6 EXPERIMENT RESULTS [\%]

\begin{tabular}{l|ccc|c}
\hline Language & Correct & Semi-correct & Erroneous & Precision \\
\hline Chinese & 25.9 & 17.0 & 57.1 & 42.9 \\
English & 39.1 & 14.2 & 46.7 & 53.3 \\
French & 33.9 & 9.9 & 56.1 & 43.9 \\
Japanese & 31.7 & 16.2 & 52.1 & 47.9 \\
Korean & 34.4 & 23.1 & 42.6 & 57.5 \\
\hline Mean & 33.0 & 16.1 & 50.9 & 49.1 \\
\hline \multicolumn{5}{c}{ Baseline* } \\
Chinese & $25.6(16.0)$ & $13.6(30.4)$ & $4.4(10.0)$ & $39.2(46.4)$ \\
English & $4.0(8.4)$ & $16.0(53.2)$ & $15.2(3.2)$ & $20.0(61.6)$ \\
German** & $17.6(19.2)$ & $19.6(20.0)$ & $13.6(10.0)$ & $37.2(39.2)$ \\
Japanese & $14.6(38.2)$ & $2.5(13.5)$ & $8.9(22.3)$ & $17.1(51.7)$ \\
\hline Mean & $15.4(20.4)$ & $12.9(29.3)$ & $10.5(11.4)$ & $28.3(49.7)$ \\
* Responses generated by the genetic algorithm part of the system \\
** Baseline experiment has also been carried out in German.
\end{tabular}

that the baseline algorithm is more suited to some languages than others.

We also asked each subject for their impressions after completing the experiment. Some users were impressed by interesting responses or somewhat strange responses, whereas others were impressed by the fact that the system asks some questions by itself.

However, the system can still only handle simple input such as greetings and simple questions about the system's personal information or tastes. Nevertheless, the system learns from user input in order to be able to reply with a greater variety of output the longer the user chats with it.

Contrary to the baseline, the proposed system does not only look for rules that match the input, but deconstructs the input into different parts to access all the related terms of the sentence, and finally chooses an output. For example, if the input contains the word "apple", since "apple" is related to "fruit", the system can respond using the word "fruit" instead of "apple". This behavior is very useful for handling problems related to synonyms and paraphrases. That is why we consider the graph structure suited to handle relation between words and to select the best response to an input.

1) Used resources: The baseline uses a morphological analysis tool and an ELIZA-like system, both of which are language-specific. In particular, morphological analysis tools use many language-dependent resources such as dictionaries and grammatical rules. However, the other parts of the output generation do not depend on language. Consequently, the system can be adapted to other languages with a minimal amount of work for any language for which such tools are provided.
However, if one of these tools is not available, the adaptation task becomes much more complicated.

In comparison, the proposed approach only needs target language samples to be trained and then be able to handle a dialogue in the targeted language. In addition, certain processing steps such as the splitting parameter and the removal of capital letters have been added to improve the system's speed, but these are not necessary for the functioning of the system.

To achieve a fully end-to-end language-agnostic dialogue system, it is possible to start the system without any knowledge and to allow it to acquire knowledge from the user input. However, in this case the supervising process can be annoying for the user. A better method would be to make the system assist with a dialogue between two humans and acquire knowledge in a similar way to a child hearing people around him and finally becoming able to speak. The dialogue samples used can be considered to be dialogue heard by the system during its "childhood".

2) Differences between languages: The system's behavior is quite similar in all the tested languages. However, depending on the language, the system tends to answer with a question or to match the wrong knowledge. Especially in French, questions that contain "est-ce que" are often replied to with another similar question. Substitution replacing links are made in order to avoid this kind of behavior. However, the construction of this kind of question is not the most frequent in the corpus; as a result, it is hard for the system to acquire the correct substitution replacing links and to decrease the score of the question's nodes when the input is also a question. 


\section{FUTURE WORK}

In order to improve the quality of the dialogue, we consider that link characteristics could be adjusted to make the system firstly answer the question, and then secondly ask questions related to the same subject more often. However, since setting an adequate value to each link requires a lot of time, this task would be better conducted automatically using a learning algorithm such as reinforcement learning [40], which is easy to implement in a dialogue system using user feedback to evaluate the system output and the used links.

Although the clustering process is working, the precision is still not satisfying, especially in language using long strings. We consider implementing more restrictions to nodes in the same cluster, such as being placed before the same node like a space in English or French. In the current system, some cluster links are set between words and parts of the same word such as "chocolate" and "chocola". If the system checks nodes around the target nodes, it can select only nodes which are used around the same nodes. For example, nodes "chocolate" and "vanilla" from strings such as "I like chocolate" and "you like vanilla" are both around the node "you" and the end of the sentence, but "chocola" is not at the end of the sentence and will not be put in the same cluster.

On another hand, the system can be set up to keep active nodes longer to use them as context. Concretely, the score of previously used nodes is kept upper zero to use them in priority for new output generation. For example, if the user inputs "I like fruits" and then "How about you?", the system will use nodes related to previous input in priority and, for example, output "I like vegetables" rather than "I am fine".

In addition, it is easy to improve the system to add other kinds of links and inputs. For example, the system can handle any kind of tags representing nonverbal data such as emotions or environmental context, in order to be improved into a multimodal dialogue system. By extension, the system can use this new data to check the coherence of the output and proceed to a logical reflection. For instance, we will add emotional nodes [41] to the graph in order to be able to generate output in function of the related emotions. As an extension, since the same graph can contain any meta-data, we will also implement more complex coherence checking using a system internal state. Each node can be related to a state, and the system can compare this state with its own state and check the coherence of the output.

Furthermore, sharing knowledge between several users [36] would help the system to acquire many different kinds of knowledge directly from the users in order to facilitate the language acquisition task.

\section{CONCLUSION}

In this paper, we used a unique language independent algorithm to provide a real-time dialogue agent to the user. We carried out experiments in Chinese, English, French, Japanese and Korean and obtained similar results in all these languages. Moreover, the precision obtained is superior to the baseline if we do not count ELIZA's responses. However, the results have to be improved to achieve its different objectives.

The baseline system handles several languages; however it needs to be adapted to each one. In contrast, the proposed system needs no special work to be adapted to another language. For example, we can input into the system both Chinese and Japanese training samples at the same time, and the system will be able to output Chinese as well as Japanese sentences.

In future, we consider that most natural language processing tools such as morphological analysis tools and partof-speech tagging tools will be able to handle any kind of language using statistical methods that are not language-dependent. Therefore, these tools could be used to improve this system's performance. However, while external tools have many advantages, they often limit the flexibility of a system and make it heavier. Moreover, it is hard to conserve knowledge consistency between all the different tools. For example, the system has no reason to know that "high school" is composed of two words if "high" was always associated with "school" within all the knowledge it has acquired, but external tools could separate them.

Moreover, to be able to handle more numerous useful nodes without generating too many useless links and nodes, the system can "forget" the latter. This 
can be done in a similar way to humans who forget wrong or non-meaningful information. As a result, the system will not require more processing than system using external resources which contain only meaningful data.

In addition, the system acquires knowledge from samples and from user inputs, and as a result can learn language-specific expressions. However, since the system cannot use language-specific operations to analyze the inputs, it needs more knowledge to acquire them in a statistical way. For example, by using part-of-speech tagging it is possible to give more importance to nouns (such as "city") than to prepositions (such as "to"), but since a languageindependent system cannot use part-of-speech tagging, it has to check the frequency of each word in a set of sentences to determine their importance. The system needs to look at the context of each node to use them correctly, while a language-specific system can use external tools to check the use of each node. Consequently, the proposed method requires many environmental information to select the appropriate output.

Finally, since the system does not use any grammarspecific processing, simple sentences, complex sentences and compound sentences are all processed similarly. That is why we consider the proposed system structure can be used to generate more complex inputs that other dialogue agents cannot handle correctly.

\section{References}

[ 1 ] Richard S. Wallace. 2009. The Anatomy of A.L.I.C.E., pp.181-210. Parsing the Turing Test.

[2] Jiyou Jia. 2009. CSIEC:A computer assisted English learning chatbot based on textual knowledge and reasoning, pp.249-255. Knowledge-Based Systems 22 (4). Elsevier.

[ 3 ] Shinsuke Higuchi and Rafal Rzepka and Kenji Araki. 2009. A casual conversation system using modality and word associations retrieved from the web, pp.181210. Parsing the Turing Test.

[4] Joseph Weizenbaum. 1966. ELIZA-A Computer Program For the Study of Natural Language Communication Between Man And Machine, pp.3645. Communications of the ACM 9 (1). Association for Computing Machinery.

[ 5 ] Ridong Jiang, Rafael E. Banchs, Seokhwan Kim, Kheng Hui Yeo, Arthur Niswar and Haizhou Li. 2014. Webbased Multimodal Multi-domain Spoken Dialogue System. Proceedings of 5 th International Workshop on Spoken Dialog Systems.

[6] Arnaud Jordan and Kenji Araki. 2013. Spoken Dialog Processing for Acquiring Taste and Knowledge. Proceedings of PACLING2013.

[ 7 ] Miki Ueno, Naoki Mori and Keinosuke Matsumoto. 2010. Novel chatterbot system utilizing web information, pp.605-612. Distributed Computing and Artificial Intelligence. Springer.

[8] Kurohashi and Kawahara lab. 2012. Japanese Morphological Analysis System JUMAN version 7. Department of Intelligence Science and Technology, Graduate School of Informatics, Kyoto University.

[9] Ryan T. McDonald, Joakim Nivre, Yvonne QuirmbachBrundage, Yoav Goldberg, Dipanjan Das, Kuzman Ganchev, Keith B. Hall, Slav Petrov, Hao Zhang, Oscar Täckström and others. 2013. Universal Dependency Annotation for Multilingual Parsing, pp.92-97. Proceedings of ACL2013.

[10] Sabine Buchholz and Erwin Marsi. 2006. CoNLL$X$ shared task on multilingual dependency parsing, pp. 149-164. Proceedings of the Tenth Conference on Computational Natural Language Learning. Association for Computational Linguistics.

[11] Christopher D. Manning and Hinrich Schutze. 1999. Foundations of Statistical Natural Language Processing. MIT Press.

[12] Yoon Kim. 2014. Convolutional neural networks for sentence classification. arXiv preprint arXiv:1408.5882.

[13] Lawrence R. Rabiner. 1989. A tutorial on hidden Markov models and selected applications in speech recognition, pp.257-286. Proceedings of the IEEE (77 2). IEEE

[14] Taku Kudo, Yuji Matsumoto. 2003. Fast Methods for Kernel-Based Text Analysis, pp.24-31. Proceedings of ACL2003.

[15] Sergey Brin and Lawrence Page. 1998. The anatomy of a large-scale hypertextual Web search engine, pp.107-117. Computer networks and ISDN systemsVolume 30. Elsevier.

[16] Jeesoo Bang, Hyungjong Noh, Yonghee Kim and Gary Geunbae Lee. 2015. Example-based chat-oriented dialogue system with personalized long-term memory, pp.238-243. 2015 International Conference onBig Data and Smart Computing (BigComp). IEEE.

[17] George A. Miller. nov. 1995. WordNet: A Lexical Database for English, volume 38, pp.39-41. Commun. ACM. ACM.

[18] Arnaud Jordan and Kenji Araki. 2014. A Framework for Multilingual Real-time Spoken Dialogue Agents, pp.24-29. Proceedings of iCAST2014.

[19] Howard C. Warren. 1921. A History Of The Association Psychology. Charles Scribner's Sons.

[20] Paul Ekman. 1972. Universals and Cultural Differences in Facial Expression of Emotion, pp. 207-283. J. Cole ed. Nebraska Symposium on Motivation. University of Nebraska Press.

[21] Beate Dorow and Dominic Widdows. 2003. Discovering corpus-specific word senses, pp.79-82. Proceedings of the tenth conference on European chapter of the 
Association for Computational Linguistics-Volume 2. Association for Computational Linguistics.

[22] David Hope and Bill Keller. 2013. MaxMax:a graphbased soft clustering algorithm applied to word sense induction, pp.368-381. Computational Linguistics and Intelligent Text Processing. Springer.

[23] David Hope and Bill Keller. 2013. UoS:A GraphBased System for Graded Word Sense Induction, pp.689-694. Second Joint Conference on Lexical and Computational Semantics (2). Proceedings of the Seventh International Workshop on Semantic Evaluation (SemEval 2013).

[24] Amita Jain, Devendra K. Tayal and Sudesh Yadav. 2014. Retrieving web search results using Max-Max soft clustering for Hindi query, pp.1-12. International Journal of System Assurance Engineering and Management. Springer.

[25] Yuji Matsumoto, Akira Kitauchi, Tatsuo Yamashita, Yoshitaka Hirano, Hiroshi Matsuda, Kazuma Takaoka and Masayuki Asahara. 2002 Morphological Analysis System ChaSen version 2.2. 9 Manual. Nara Institute of Science and Technology.

[26] Daichi Mochihashi, Takeshi Yamada and Naonori Ueda. mar. 2009. Bayesian Unsupervised Word Segmentation with Hierarchical Language Modeling, pp.49-56. IPSJ SIG Notes 2009 (36). Information Processing Society of Japan (IPSJ) .

[27] Luca Gammaitoni, Peter Hänggi, Peer Jung and Fabio Marchesoni. 1998. Stochastic resonance, volume 70, pp.223. Reviews of modern physics. American Physical Society (APS).

[28] Gerard Salton and Michael J. McGill. 1986. Introduction to modern information retrieval. PMcGraw-Hill, Inc.

[29] Masashi Takeuchi, Norihide Kitaoka and Seiichi Nakagawa. 2004. A spoken dialog system activating the natural response timing using prosodic and linguistic information for chat-like conversation, pp.87-92. IPSJ SIG Notes 2004 (15). Information Processing Society of Japan (IPSJ) .

[30] Richard S. Wallace. 2003. The elements of AIML style. Alice AI Foundation.

[31] George Lakoff and Mark Johnson. 1989. The metaphorical structure of the human conceptual system, volume 4-2, pp.195-208. Elsevier.

[32] Kenji Araki and Michitomo Kuroda. 2006. Generality of spoken dialogue system using SeGA-IL for different languages, pp.72-77. Proceedings of the Second IASTED. Computational Intelligence.

[33] Geoffrey Hinton, Li Deng, Dong Yu, George E. Dahl, Abdel-rahman Mohamed, Navdeep Jaitly, Andrew
Senior, Vincent Vanhoucke, Patrick Nguyen, Tara N. Sainath and others. 2012. Deep neural networks for acoustic modeling in speech recognition: The shared views of four research groups, volume 29-6, pp.8297. Signal Processing Magazine. IEEE.

[34] Satoshi Sekine and Ralph Grishman. 1995. A Corpusbased Probalistic Grammar with Only Two NonTerminals. Proceedings of the Fourth International Workshop on Parsing Technologies.

[35] Hua-Ping Zhang, Hong-Kui Yu, De-Yi Xiong and Qun Liu. 2003. Q.HHMM-base Chinese lexical analyzer ICTCLAS, volume 17, pp.184-187. Proceedings of the Second SIGHAN Workshop on Chinese Language Processing.

[36] Arnaud Jordan and Kenji Araki. 2013. Comparison of two Knowledge Treatments for Questions Answering pp.55-62. Proceedings of SNLP2013.

[37] Sangwon Park, DongHyun Choi, Eun-kyung Kim, KeySun Choi. 2010. A Plug-In Component-based Korean Morphological Analyzer, pp.197-201. Proceedings of HCLT 2010.

[38] Tim Menzies, Alex Dekhtyar, Justin Distefano and Jeremy Greenwald. 2007. Problems with Precision: A Response to "Comments on 'Data Mining Static Code Attributes to Learn Defect Predictors'”, volume 339, pp.637-640. IEEE Trans. Softw. Eng. IEEE Press.

[39] Kenji Araki and Michitomo Kuroda. Jan. 2007. Evaluation of Generality of SeGA-ILSD for a Chat Using Different Languages, volume 2007, pp.79-85. IPSJ SIG Notes 2007 (7). Information Processing Society of Japan (IPSJ) .

[40] Leslie Pack Kaelbling, Michael L. Littman and Andrew W. Moore. 1996. Reinforcement learning: A survey. arXiv preprint cs/9605103.

[41] Michal Ptaszynski, Pawel Dybala, Rafal Rzepka and Kenji Araki. 2008. Effective analysis of emotiveness in utterances based on features of lexical and nonlexical layer of speech, pp.171-174. Proceedings of the Fourteenth Annual Meeting of the Association for Natural Language Processing.

（2015年10月 5 日 受付） （2015年11月27日 採録）

[Contact Address ]

Graduate School of Information, Science and Technology Hokkaido University, Sapporo, Japan

Arnaud JORDAN

TEL : +81-11-706-7389

FAX : +81-11-709-6277

E-mail : arnaud@media.eng.hokudai.ac.jp 


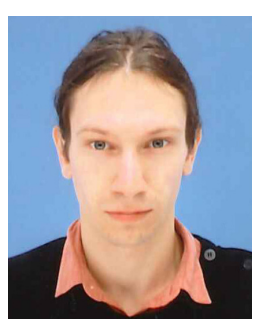

\section{Arnaud JORDAN [non-member]}

He received master's dregree from ENSISA Engineering School of the University of Haute-Alsace (UHA) in 2012. Currently, he is doctoral course student at Graduate School of Information Science and Technology, Hokkaido University, Japan. His research interests include natural language processing, language acquisition, and autonomous systems.

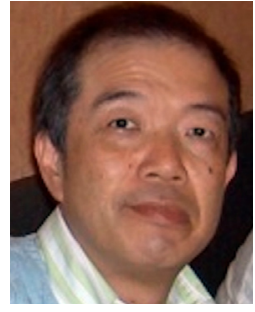

\section{Kenji ARAKI [member]}

Kenji Araki received the BE, ME, and $\mathrm{PhD}$ degrees in electronics engineering from Hokkaido University, Sapporo, Japan, in 1982, 1985, and 1988, respectively. In April 1988, he joined Hokkai Gakuen University, Sapporo, Japan, where he was a professor. He joined Hokkaido University in 1998 as an associate professor in the Division of Electronics and Information Engineering and became a professor in 2002 . Presently, he is a professor in the Division of Media and Network Technologies at Hokkaido University. His research interests include natural language processing, spoken dialogue processing, machine translation, and language acquisition. 